John Carroll University

Carroll Collected

$12-2017$

\title{
Motivational interviewing and dual diagnosis clients: Enhancing self-efficacy and treatment completion
}

Martina S. Moore

John Carroll University, mmoore@jcu.edu

Brende Flamez

G. Mihalyi Szirony

Follow this and additional works at: https://collected.jcu.edu/fac_bib_2017

Part of the Counseling Commons

\section{Recommended Citation}

Moore, Martina S.; Flamez, Brende; and Szirony, G. Mihalyi, "Motivational interviewing and dual diagnosis clients: Enhancing selfefficacy and treatment completion" (2017). 2017 Faculty Bibliography. 49.

https://collected.jcu.edu/fac_bib_2017/49 


\title{
Motivational interviewing and dual diagnosis clients: Enhancing self-efficacy and treatment completion
}

\author{
Martina Moore ${ }^{\mathrm{a}}$, Brandé Flamez ${ }^{\mathrm{b}}$, and G. Mihalyi Szirony ${ }^{\mathrm{c}}$ \\ a Mental Health Counseling Department, John Carroll University, University Heights, Ohio, USA; ${ }^{b}$ Department of Counseling and Special Populations, \\ Lamar University, Beaumont, Texas, USA; 'SChool of Counseling, Walden University, Minneapolis, Minnesota, USA
}

\begin{abstract}
This study explored treatment completion and self-efficacy of dual-diagnosis clients receiving cognitive behavioral therapy (CBT) and a combination of CBT with motivational interviewing (MI) in an intensiveoutpatient setting. Results indicated that MI was associated with increased self-efficacy and treatment completion.
\end{abstract}

Drug overdose is among the leading causes of death in the United States (Winstanley et al., 2012). Between the years of 2010 and 2012, approximately 15.2 million females and 19.8 million males used illicit drugs in the United States (Diaz, Horton, \& Weiner, 2012). Researchers have reported that over $50 \%$ of individuals who abused drugs had a comorbid mental health disorder (Diaz et al., 2012), and approximately 75\% of individuals who were diagnosed with a substance use disorder (SUD) had a concomitant, treatable mental health diagnosis in their lifetime (Cridland, Deane, Hsu, \& Kelly, 2012). The National Survey of Substance Abuse Treatment Services (Substance Abuse and Mental Health Services Administration, Center for Behavioral Health Statistics and Quality, 2014) reported that $43 \%$ of all clients treated for substance abuse had a co-occurring mental health disorder. The co-presentation of mental health disorders and SUDs, hereafter referred to as dual diagnosis (DD), is prevalent in the United States. The Substance Abuse and Mental Health Administration (United States Department of Substance Abuse and Mental Health Administration (SAMHSA), 2011) reported that more than 6.8 million adults hold a DD.

The treatment of the DD population presents unique challenges to payers and clinicians. SAMHSA (United States Department of Substance Abuse and Mental Health Administration (SAMHSA), 2014) reported that the cost to treat individuals with DD was over $\$ 100$ billion annually. Clinicians are faced with various obstacles in treating this population, including finding effective ways to engage and retain DD clients in treatment. The presence of comorbid SUDs and mental health disorders often exacerbates the effects of both diagnoses (Westra, Aviram, \& Doell, 2011) and has been historically linked with poor treatment outcomes (Cridland et al., 2012).

While clients may present with a variety of combinations of mental health disorders and SUDs, Cridland et al. (2012) reported that the most prevalent DD combination included a mood disorder and alcohol use disorder. The co-presentation of alcohol use and a mood disorder is significant, as an interrelation between suicide attempts, depression, and alcohol addiction was reported (Lyne, O'Donoghue, Clancy, \& O'Gara, 2011). Researchers also found that the combination of substance abuse and mood disorders significantly increased the risk for completed suicide (Lyne et al., 2011). Clients with DD reported higher frequencies of attempted suicide, resulting in recurring hospital stays (Błachut, Badura-Brzoza, Jarzab, Gorczyca, \& Hese, 2013).

Because of the risks and increased symptom severity associated with holding a DD, these clients have often required extended inpatient treatment (Cridland et al., 2012). Those with DD also experienced higher incidences of inpatient treatment with longer hospital stays for multiple treatments (Benaiges, Prat, \& Adan, 2012). When comparing DD clients with those diagnosed with only a SUD, Cridland et al. (2012) found that DD clients typically had higher relapse rates and poorer treatment outcomes. These outcomes were cited as being related to noncompliance with treatment recommendations. Additionally, poor treatment outcomes indicated that those with DD experienced higher rates of relapse that were associated with increased legal involvement and recidivism when compared to those diagnosed with a standalone SUD.

\section{Barriers to treatment completion}

According to Tate et al. (2011), there was a significant treatment dropout rate among substance-abusing clients. Dropout rates have been reported as high as $82 \%$ for intensive outpatient treatment. According to a systematic review by Brorson, Arnevik, Rand-Hendriksen, and Duckert (2013), it is more common for patients to drop out of treatment than to complete. From their review, $21.5-43 \%$ of individuals in detoxification drop out, $3-50 \%$ for outpatient treatment 
leave, up to $57 \%$ in inpatient treatment terminate, and up to $67.7 \%$ fail to complete substitution treatment programs. Because of complications when treating clients with co-occurring disorders in the United States, such as demographics, social structure, and cognitive functioning, treatment outcomes remained poor (Sterling, Chi, \& Hinman, 2011). Treatment for the DD population is increasingly problematic for practitioners, and challenges remain when assisting DD clients in successfully completing treatment (Błachut et al., 2013).

According to Patterson, Wolf, and Buckingham (2010), DD clients experienced multiple barriers that affected treatment outcomes. The lack of an integrated treatment model for concurrent treatment of both diagnoses has historically presented a barrier for DD clients. For instance, when clients warranted dual treatment for $\mathrm{DD}$, treatment facilities offered treatment for one disorder, requiring clients to visit another facility for treatment of the other diagnosis. Further, Patterson et al. (2010) found that DD clients did not follow through with the treatment of a second diagnoses, which remained untreated.

The DD population also has experienced barriers associated with having limited social support from family (Horsfall, Cleary, Hunt, \& Walter, 2009). Many DD clients participate in maladaptive behaviors and engage in multiple treatment episodes, causing family members to withdraw or experience burnout (Horsfall et al., 2009). Clients with DD also experienced lack of supportive sober housing and limited employment options. In order to improve treatment rates for this population, a combination of person-centered approaches and cognitive therapy offered from a multi-disciplinary team was suggested (Horsfall et al., 2009).

\section{Self-efficacy and treatment completion}

Self-efficacy encompasses a person's belief about his or her ability to make behavioral changes and is required for successful goal attainment (Greenfield, Venner, Kelly, Slaymaker, \& Bryan, 2012). Researchers found an association between increasing self-efficacy and accomplishing goals, such as behavioral change treatment, for DD clients (Stein, Zane, \& Grella, 2012). The results further showed that by increasing selfefficacy, clients were more likely to complete treatment. Behavioral self-efficacy includes assessing the way people think, behave, and feel about changing a behavior. For example, Greenfield et al. (2012) reported that a client with high levels of behavioral self-efficacy also held a high level of confidence in his or her ability to complete treatment goals and objectives. These results also indicated that clients with high levels of self-efficacy had high levels of belief in their ability to stop the use of addictive substances. The effects of self-efficacy on goal attainment were also demonstrated in a study by Luszczynska, Benight, and Ciesak (2009) that found that substance-abusing clients with high levels of behavioral self-efficacy also held a stronger commitment to the plan of change. Additionally, clients who reported high levels of behavioral self-efficacy recovered from relapse quicker and remained committed to the change process.

In contrast, Luszczynska et al. (2009) found that clients with low levels of behavioral self-efficacy displayed low levels of confidence in their abilities to achieve treatment goals (i.e., discontinue use) and objectives. These results further indicated that a client's ability reduced when trying to make necessary behavioral changes related to ending drug addictions (Luszczynska et al., 2009). Stein et al. (2012) further demonstrated this finding, reporting that clients with low levels of behavioral self-efficacy who used tobacco did not attempt behavioral change strategies and often avoided changes.

As the literature has reported, low levels of self-efficacy are often associated with continued drug use behavior; however, when exposed to self-efficacy enhancing programs, clients appeared more likely to remain in drug cessation treatment (Elfeddali, Bolman, Candel, Wiers, \& De Vries, 2012). The researchers further stated that exposing clients to psychological treatments such as motivational interviewing (MI; Elfeddali et al., 2012) was beneficial for increasing clients' confidence to remain in treatment. The study results also revealed that clients who completely stopped using drugs experienced high levels of self-efficacy to remain in treatment during the research period, while those using drugs intermittently experienced significantly low levels of self-efficacy to remain in treatment.

The study results indicated that self-efficacy significantly increased through complete abstinence and not by gradual cessation of drug use. In contrast, Mason, Deane, Kelly, and Crowe (2009) found that clients with low levels of self-efficacy during drug use treatment experienced increased depression and stress. The results suggested that increasing self-efficacy benefits increasing behavioral change. For instance, Mason et al. (2009) found that introducing spirituality to drugaddicted clients as a coping strategy increased self-efficacy and also reduced cravings and behavior relapse. The study indicated that using interventions provided better control over situational confidence for DD clients because of increased self-efficacy.

\section{Cognitive behavioral therapy and MI}

Cognitive behavioral therapy (CBT) has been identified as a leading treatment method used with the substance abusing population (Aviram \& Westra, 2011). High completion rates of CBT treatment benefit positive social change by decreasing DD clients' relapse rates that, in turn, decrease early mortality. Furthermore, abstaining from substance abuse is associated with improved mental health and occupational functioning for DD clients. These clients also experience less legal involvement, a decrease in medical complications related to drug abuse, and overall improvement in quality of life.

Flynn (2011) reported that CBT (Butler, Chapman, Forman, \& Beck, 2006) has shown efficacy for reducing suicide and depression in many clients, issues that have been cited for their comorbidity with SUDs. However, a major criticism of CBT has been that a significant number of substance abusing clients have high rates of treatment recidivism. According to Westra, Aviram, Connors, Kertes, and Ahmed (2012), theoretical orientations that engaged CBT reported high levels of client resistance. For instance, clients argued with the therapist about completing assignments and were 
resistant toward recommendations, all leading to high rates of recidivism. Further, the authors argued that outcomes suggested that high levels of client resistance correlated with failure to complete treatment.

MI has been suggested as an augmentation to CBT to address issues related to client resistance (Burke, 2011). MI is a collaborative conversation, an approach that helps clients with the primary purpose of strengthening a person's motivation for change and, in particular, as Miller and Rollnick (2013) put it, “...a person's own motivation” (p. 12). Techniques can include expressing empathy through reflective, high-quality listening, developing discrepancies between client's goals and their current behavior, rolling with a client's resistance rather than opposing it, using efficacious language, among others. Depending on the needs of the client, each technique can be utilized independently. For instance, clinicians might use the empathy technique to help clients feel less judged and promote feelings of comfort that allows the client to communicate openly with the professional (Iarussi, Tyler, Littlebear, \& Hinkle, 2013). Professionals use the discrepancy technique to help clients explore the logic of behavior compared to a desired outcome. Rolling with resistance techniques benefit both clients and professionals, and are a way to avoid viewing resistance to change as a barrier, and rather allow clients to rediscover discrepancies contributing to the resistance. Additionally, as clients begin to struggle with their abilities to change behaviors, utilizing efficacious language can help to support self-efficacy and optimism. Finally, utilizing reflective listening skills throughout the process is a technique that improves communication and reduces misunderstanding between the client and clinician. The use of MI techniques and skills helps to accomplish the overall goal of expressing unconditional positive regard toward the client and allows the professional to attend to cues not communicated (Westra et al., 2011).

There was an abundance of literature on the utilization of CBT with the substance abusing population (Aviram \& Westra, 2011; Najavits \& Hien, 2013; Windsor, Jemal, \& Alessi, 2015; Wolff, Frueh, Shi, \& Schumann, 2012); however, very few studies explored the benefits of utilizing MI as an ongoing treatment to increase treatment completion rates. The lack of research in this area presented a problem for understanding influences that led to increasing clients' treatment completion rates. A gap in the literature exists and there has been a lack of research on the effectiveness of MI as an ongoing adjunct treatment to CBT when treating individuals with DD in intensive-outpatient (IOP) treatment. Closing the gap provides advantages for assisting mental health practitioners in promoting completion rates for clients who are engaged in CBT programming.

\section{The current investigation}

The purpose of this study was to investigate whether there was a statistically significant difference in IOP completion rates of DD clients who received an $\mathrm{MI}$ and CBT treatment when compared to those receiving CBT alone. This study also examined whether there was a significant difference in preand post-intervention behavioral self-efficacy to complete treatment. A quantitative methodology was used in the current study, along with a quasi-experimental design to explore whether clients' IOP treatment completion rates statistically significantly improved after receiving MI. The independent variables in this study were MI and behavioral self-efficacy, and the dependent variable was CBT completion rates. An additional goal of conducting this study was to investigate whether MI and CBT treatment statistically significantly increased clients' self-efficacy. The results of this study may provide valuable directions for further exploring the use of MI in conjunction with CBT as an evidence-based practice for practitioners to utilize in the treatment of DD clients.

\section{Method}

\section{Sampling procedures}

The sample included observations from secondary data for this study. The sample was chosen to represent three study groups that are (1) a comparison group receiving CBT treatment alone; (2) a treatment group receiving $\mathrm{MI}$ and $\mathrm{CBT}$ treatment; and (3) a treatment group receiving $\mathrm{MI}$ and $\mathrm{CBT}$, where self-efficacy was recorded pre- and post-MI. A random generator table was used to randomly generate 280 cases from a pool of available records $(N=5750)$. The researchers chose the RAND function in Microsoft Excel 2010 in an effort to reduce bias and provide representation of all levels of the phenomenon (Chacón, Alvarado, \& Santisteban, 2011). According to a $\mathrm{G}^{*}$ Power analysis, with a medium effect size, an alpha level of .05, and a power level of .80, $N=280$. The current sample size $(N=307)$ more than satisfied the minimum sample size required for the study based upon the $\mathrm{G}^{\star}$ Power analysis. Data collection occurred by randomly choosing samples for analysis.

\section{Participants}

The population of individuals studied comprised DD clients within the United States. According to SAMHSA (2014), the DD population represents more than $40 \%$ of clients receiving drug and alcohol treatment. The researchers intended to select a sample for this study that would be representative of the population of DD clients in the United States so that the results of the current study could be helpful in understanding the efficacy of $\mathrm{MI}$ as an intervention for increasing CBT completion rates.

Participants included DD clients who received IOP treatment from a treatment center located in northeast Ohio. Clients in the region were an appropriate representation of people who suffer from mental health disorders and were representative of the US mental health disorder population. Results of at least two studies (Forand \& DeRubeis, 2013; Winstanley et al., 2012) indicated that studying a sample from the northeast Ohio region was beneficial for understanding the effects of MI on completing CBT for DD clients in the region.

The randomly selected sample included 210 males that made up $68 \%$ of the sample and 97 females that made up the rest of the sample. The ethnic makeup of the group was 
analyzed and it was found that African Americans $(n=152)$ made up the largest proportion (49\%) of the sample and Whites $(n=140)$ made up the second largest proportion $(46 \%)$ of the group. People who identified as multi-racial $(n=13)$ and Hispanics $(n=2)$ made up the rest of the sample.

Most participants had a high school diploma or equivalent ( $n=228)$ and made up $74 \%$ of the group. The second highest proportion of the group were people with less than a high school education $(n=51)$ who contributed to $17 \%$ of the sample. Clients who either completed or attended college made up the rest of the sample. The results indicated that approximately 1 in 10 people who engaged in drug use had some college education or higher and approximately 1 in 5 had less than a high school education.

Alcohol was the most popular drug of choice used among clients $(n=155)$ and comprised $51 \%$ of the sample. The results demonstrated that 5 in 10 people required treatment for alcohol use. Marijuana was the next highest drug of choice $(n=70)$ and is $23 \%$ of the sample, indicating that for clients who use drugs, approximately one in four prefer to use marijuana. The results showed that approximately 1 in 10 used crack/cocaine $(n=33)$ and the rate is similar to heroin/opioids/prescription category of drugs.

\section{Results}

A Chi-square analysis was conducted to determine if there was a significant difference in rate of successful completion based on receiving $\mathrm{MI}$ and $\mathrm{CBT}$ as compared to receiving
CBT alone. The test results were considered to be normally distributed based on the sample size $(N=307)$. The statistical assumptions did not indicate a statistical violation.

Those who completed CBT treatment and did not receive MI comprised $26 \%$ of the overall treatment group, while participants who completed CBT treatment and received MI made up the remaining $74 \%$ of the group. Participants who did not complete treatment $(n=61)$ were split into two groups. Individuals who received MI and CBT $(n=31)$ comprised roughly $51 \%$ of all who did not complete treatment. Figure 1 shows the frequencies for each group. The Chisquare results showed an association between receiving $\mathrm{MI}$ and CBT as compared to receiving CBT alone $\chi^{2}(1$, $N=307)=11.85, p<.01, V=.20$. The results demonstrated a significant difference in the rate of successful completion of treatment between all groups based on group and treatment assignment.

The mean difference between pre-self-efficacy scores $(M=7.68, \mathrm{SD}=2.55)$ and post-self-efficacy scores $(M=9.06, \mathrm{SD}=1.29)$ was -1.38 , indicating a $14 \%$ increase in scores for participants receiving MI treatment. The median score increased by .75 and the range decreased from 9 to 6 between the lowest and highest scores after MI treatment. The results showed that participants with low self-efficacy scores increased by $30 \%$ after receiving MI treatment.

Some clients started with a higher level of self-efficacy ( $n=26)$, but $61 \%$ of participants $(n=46)$ experienced a positive change in self-efficacy. Some change was as little as

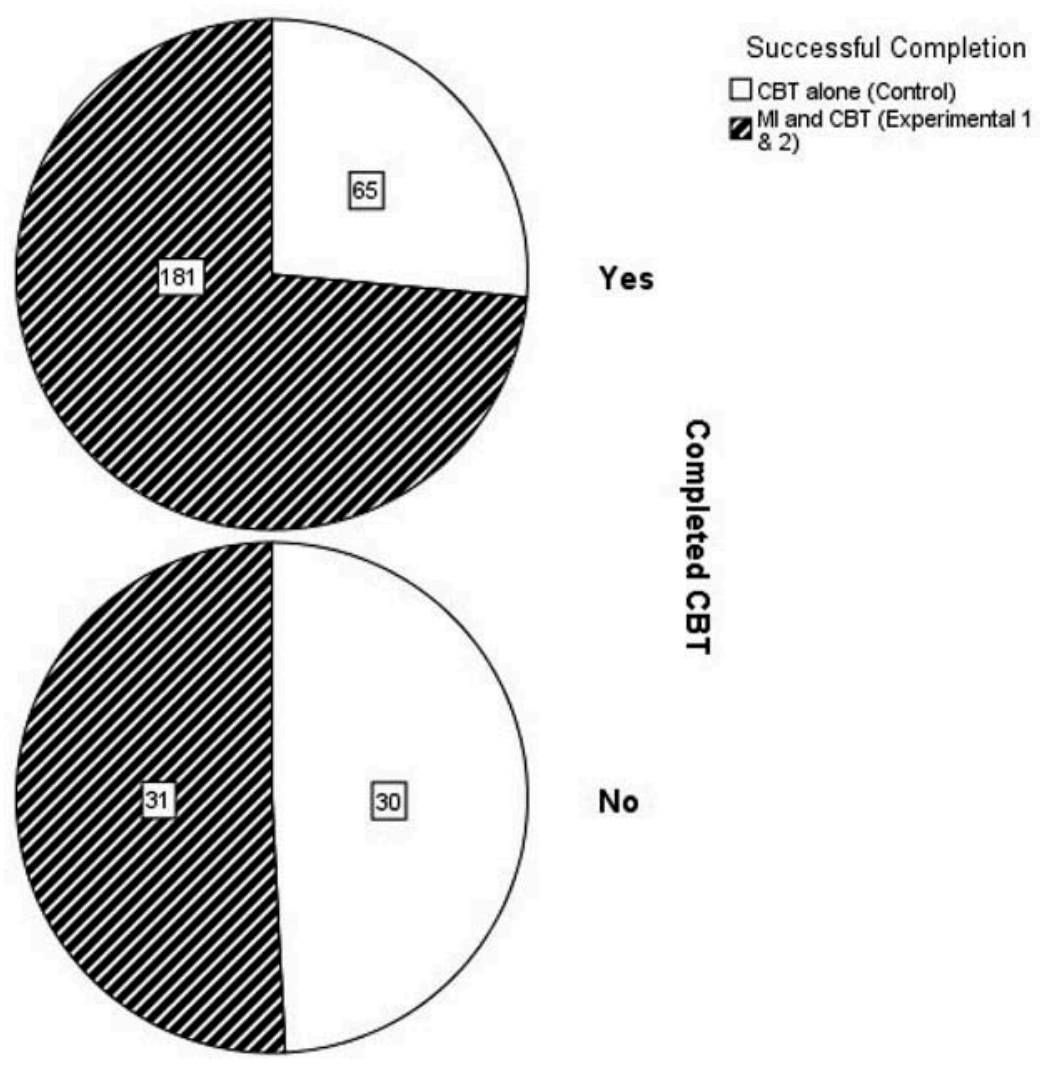

Figure 1. A pie chart showing the group frequency between participants completing treatment and the level of treatment provided. 


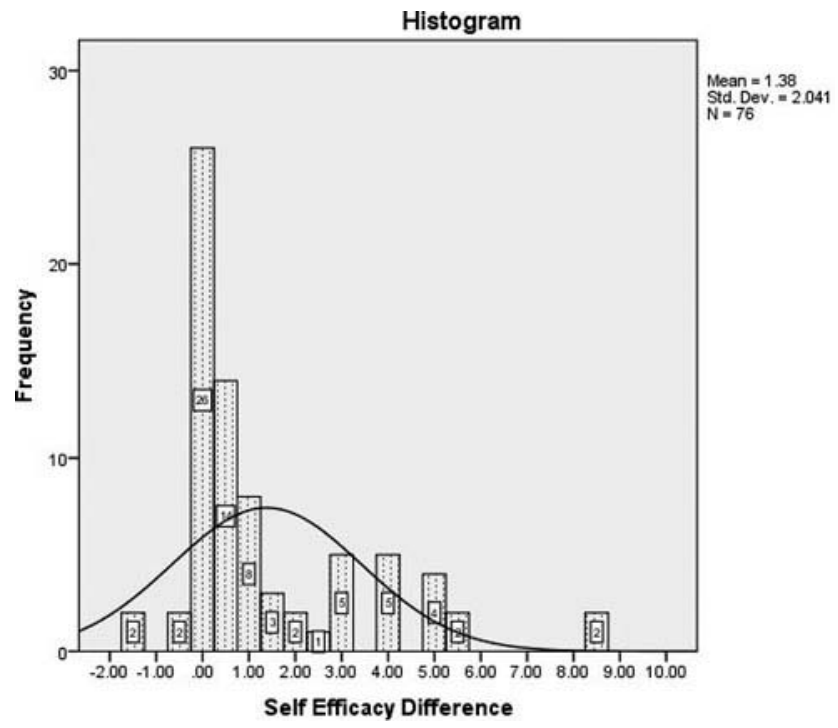

Figure 2. A histogram showing the distribution of difference scores for pre and post-self-efficacy scores.

less than $10 \%$, while others experienced as much as an $80 \%$ increase in self-efficacy to complete CBT when receiving MI. Distributions of the difference scores are included in Figure 2. The results of the paired samples $t$-test were significant $t$ $(75)=-5.91, p<.001, \eta^{2}=.32$, suggesting that a significant difference was found.

\section{Discussion}

\section{Implications}

Analyses of treatment completion rates suggested that the expected completion rate for participants who received CBT alone was $n=19$; instead, this study found that $n=30 \mathrm{did}$ not complete treatment. These results indicate that more participants in the comparison group did not complete treatment than had been expected. Similarly, fewer participants in this condition $(n=65)$ completed treatment.

In comparison, those who received MI and CBT yielded higher treatment completion rates $(n=181)$ than were expected $(n=170)$ and also discontinued treatment $(n=31)$ at rates lower than anticipated $(n=42)$. The critical Chi-square value for rejecting the null hypothesis is $\chi^{2}=3.84$. Statistical analysis produced a value $\left(\chi^{2}=11.85\right)$ higher than the critical value, indicating that the null hypothesis is rejected. The Cramer's $V$ provided a small effect size $(V=.20)$ between treatment completion and treatment level; the results were significant. A cross tabulation of group results is in Table 1 .

Analysis of self-efficacy suggests that there was a significant difference in behavioral self-efficacy to complete treatment for the experimental group after receiving $\mathrm{MI}$ in combination with CBT treatment as compared to levels assessed before receiving treatment. In addition, the $95 \%$ confidence interval of -1.85 to -.92 indicated that since there is no zero value within the interval, the populations mean difference is not likely to be no difference, less than -1.85 , or more than -.92 ,
Table 1. Descriptive results of cross tabulation between treatment completed treatment level.

\begin{tabular}{llccc}
\hline & & \multicolumn{3}{c}{ Successful completion } \\
\cline { 3 - 5 } & & CBT & MI and & \\
& alone & CBT & Total \\
\hline Completed CBT & No observed count & 30 & 31 & 61 \\
& $\quad$ Expected count & 19 & 42.1 & 61.0 \\
& $\begin{array}{l}\text { Percentage within successful } \\
\text { completion }\end{array}$ & $31.6 \%$ & $14 \%$ & $19.9 \%$ \\
& Yes count & 65 & 181 & 246 \\
& Expected count & 76.1 & 169.9 & 246.0 \\
& Percentage within successful & $68.4 \%$ & $85.4 \%$ & $80.1 \%$ \\
completion & & & \\
Total & Count & 95 & 212 & 307 \\
& $\quad$ Expected count & 95.0 & 212.0 & 307.0 \\
& Percentage within successful & $100.0 \%$ & $100.0 \%$ & $100.0 \%$ \\
& completion & & & \\
\hline
\end{tabular}

Table 2. A summary of paired samples $t$-test results.

\begin{tabular}{|c|c|c|c|c|c|c|c|c|}
\hline & \multicolumn{5}{|c|}{ Paired differences } & \multirow[b]{3}{*}{$t$} & \multirow[b]{3}{*}{$\mathrm{df}$} & \multirow[b]{3}{*}{$p$} \\
\hline & \multirow[b]{2}{*}{$M$} & \multirow[b]{2}{*}{ SD } & \multirow[b]{2}{*}{ SE } & \multicolumn{2}{|c|}{$\begin{array}{l}95 \% \mathrm{Cl} \text { of the } \\
\text { difference }\end{array}$} & & & \\
\hline & & & & Lower & Upper & & & \\
\hline $\begin{array}{l}\text { Pair } 1 \text { self-efficacy pre-MI } \\
\text { - self- }\end{array}$ & -1.38 & 2.04 & .23 & -1.85 & -.92 & -5.91 & 75 & .001 \\
\hline
\end{tabular}

supporting a rejection of the null hypothesis. A summary of the results of this analysis can be found in Table 2.

In the early 1990s, Wierzbicki and Pekarik (1993) examined mean dropout rates in a meta-analysis of 125 studies. Nearly half of individuals who presented for therapy dropped out. In 2013, Brorson et al. found similar results. Although a wide variety of treatment settings and diagnoses were reviewed, including outpatient, inpatient, group, individual, family, and couples therapy, the impact remains significant. Economic, psychosocial, sociopolitical, and clinical factors are linked to dropout. By identifying factors associated with dropout rates, conversely, self-efficacy to remain in treatment can be salient to treatment success.

Counselors and psychotherapists, treatment centers, and referral sources alike benefit from more efficacious treatment options as well as better knowing levels of self-efficacy toward treatment completion. Awareness of self-esteem and MI can serve as a predictive factor in promoting outcome success. Treatment planning can be challenging, particularly for individuals with addictive disorders co-occurring with mental health issues, due to the complications of comorbidity, the problem is exacerbated. As a result of the present study, analysis of self-efficacy suggests that there was a significant difference in behavioral self-efficacy that could lead to increased therapy completion rates. By better preparing treatment in difficult populations of individuals who fall within this category, options expand for counselors working in the field.

\section{Limitations}

The sample size for participants in the self-efficacy group was small $(n=81)$ and reduced the power level for the results, possibly causing a Type I error, which is reporting a genuine 
effect in the study when there is not one. The overall sample size was large enough for genuinely understanding the effects of MI on completing CBT treatment, and the large sample size provided high power level for rejecting the null hypothesis when false and avoiding a Type II error. Conducting the study utilizing a quasi-experimental design contributed the low representation in the self-efficacy group. A true experimental design study would allow for random selection prior to starting the study and would mitigate for large enough group sizes with attrition for each group in the study.

\section{Directions for future research}

The current study focused on DD clients with mental health and SUDs, but future research could explore the treatment of clients with triple diagnoses, that is, comorbid presentation of SUDs, mental health disorders, and medical conditions. Durvasula and Miller (2014) found that MI was effective for treating triple diagnoses clients with CBT. Future research could include studying the effectiveness of MI when treating triple diagnoses clients with CBT including mental health disorders, SUDs, and comorbidities such as diabetes and hypertension. Studying clients with triple diagnoses including medical conditions is valuable to the profession due to the overwhelming number of clients who have a substance use and mental health disorder that exacerbates the symptoms of the medical conditions.

Self-efficacy theory was foundational in the study for testing the effectiveness of MI with DD populations. MI includes a self-efficacy component in the treatment; however, there are no known studies at this time on the effectiveness of planned behavior. According to Duncan, ForbesMcKay, and Henderson (2012), the theory of planned behavior is useful for predicting people's intentions and actual behavior. Duncan et al. (2012) stated when people intend to meet certain goals, the likelihood of meeting those goals increases due to planned behavior. The focus of future studies can include utilizing the theory of planned behavior as a pre-assessment to determine a client's desire for making a change. Clients with a low level of desire for making change are unlikely to complete CBT treatment and are likely candidates for receiving MI intervention. Clients with a high level of desire for making change are likely to complete CBT treatment and may not require a full course of MI intervention, if any at all (Duncan et al., 2012). An additional recommendation includes treatment agencies implementing an assessment or screening process that measures their clients' self-efficacy levels prior to beginning treatment. This vital information can assist treatment agencies with identifying clients' beliefs about the change process and their ability to make the needed changes toward treatment goals. In turn, this vital information will assist clinicians with developing treatment plans that are individualized and geared toward the client's needs. Finally, when treatment agencies have the knowledge of the client's selfefficacy levels, then implementation of MI with the stages of change to motivate clients to increase their self-efficacy levels can occur (Prochaska, Norcross, \& DiClemente, 2013). The increase in self-efficacy levels would assist with building a stronger therapeutic alliance and lead to higher rates of treatment completion.

\section{Conclusion}

People with low levels of internal motivation often need a cognitive boost to help complete treatment goals. There is a significant association between receiving an $\mathrm{MI}$ intervention and completing CBT treatment. MI is therefore a technique that can be useful for building the internal motivation of clients for completing goals such as CBT with the DD population. DD clients often experience treatment compliance issues and are great candidates for receiving MI techniques. Practitioners who utilize MI techniques at the beginning of treatment can help motivate a client through increased selfefficacy and a propensity to remain in treatment. Clients are more likely to complete CBT sessions when receiving motivation throughout the course of treatment. The results of the current study suggest that clients who receive treatment that incorporates MI techniques are more likely to complete treatment.

Having a treatment tool that is effective for improving DD clients' motivation for completing treatment is useful for practitioners. The results of the current study provided significant evidence to practitioners, researchers, and organizations on helping individuals with DD to complete CBT treatment. Professionals working with the DD population who received training to master the skills and abilities of MI would be in a better position to serve that population by using a tool that shown to be effective in its treatment. Organizations that provide treatment to DD populations are encouraged to promote the use of MI to help increase positive treatment outcomes, such as preparing individuals who use substances to return to society and live as productive citizens.

\section{References}

Aviram, A., \& Alice Westra, H. (2011). The impact of motivational interviewing on resistance in cognitive behavioral therapy for generalized anxiety disorder. Psychotherapy Research, 21, 698-708. doi:10.1080/10503307.2011.610832

Benaiges, I., Prat, G., \& Adan, A. (2012). Health-related quality of life in patients with dual diagnoses: Clinical correlates. Health and Quality of Life Outcomes, 10(1), 1-11. doi:10.1186/1477-7525-10-106

Błachut, M., Badura-Brzoza, K., Jarzab, M., Gorczyca, P., \& Hese, R. (2013). Dual diagnoses In psychoactive substance abusing or dependent persons. Psychiatria Polska, 47(2), 335-352. Retrieved from http://www.researchgate.net/journal/0033-2674_Psychiatria_polska.

Brorson, H. H., Arnevik, E. A., Rand-Hendriksen, K., \& Duckert, F. (2013). Drop-out from addiction treatment: A systematic review of risk factors. Clinical Psychology Review, 33(8), 1010-1024.

Burke, B. (2011). What can motivational interviewing do for you? Cognitive and Behavioral Practice, 18, 74 81. doi:10.1016/j. cbpra2009.08.004

Butler, A. C., Chapman, J. E., Forman, E. M., \& Beck, A. T. (2006). The empirical status of cognitive-behavioral therapy: A review of metaanalyses. Clinical Psychology Review, 26(1), 17-31.

Chacón, E., Alvarado, J. M., \& Santisteban, C. (2011). A simulation procedure for the generation of samples to evaluate goodness of fit indices in item response theory models. Methodology: European Journal of Research Methods for the Behavioral and Social Sciences, 7, 56-62. doi:10.1027/1614-2241/a000022 
Cridland, E., Deane, F., Hsu, C., \& Kelly, P. (2012). A comparison of treatment outcomes for individuals with substance use disorder alone and individuals with probable dual diagnoses. International Journal of Mental Health \& Addiction, 10, 670-683. doi:10.1007/s11469-011$9364 \mathrm{z}$

Diaz, N., Horton, E., \& Weiner, M. (2012). Dysthymia, major depression, and double depression among individuals receiving substance abuse treatment. Health, 4, 1229-1237. doi:10.4236/health.2012.412181

Duncan, E. M., Forbes-Mckay, K. E., \& Henderson, S. E. (2012). Alcohol use during pregnancy: An application of the theory of planned behavior. Journal of Applied Social Psychology, 42(8), 1887-1903. doi:10.1111/j.1559-1816.2012.00923.x

Durvasula, R., \& Miller, T. R. (2014). Substance abuse treatment in persons with HIV/AIDS: Challenges in managing triple diagnosis Behavioral Medicine (Washington, D.C.), 40(2), 43-52. http://doi.org/ $10.1080 / 08964289.2013 .866540$

Elfeddali, I. I., Bolman, C. C., Candel, M. M., Wiers, R. W., \& De Vries, H. H. (2012). The role of self-efficacy, recovery self-efficacy, and preparatory planning in predicting short-term smoking relapse. British Journal of Health Psychology, 17, 185-201. doi:10.1111/j.20448287.2011.02032.x

Flynn, H. A. (2011). Setting the stage for the integration of motivational interviewing with cognitive behavioral therapy in the treatment of depression. Cognitive and Behavioral Practice, 18, 46-54. doi:10.1016/j.cbpra.2009.09.0

Forand, N. R., \& DeRubeis, R. J. (2013). Pretreatment anxiety predicts patterns of change in cognitive behavioral therapy and medications for depression. Journal of Consulting and Clinical Psychology, 81, 774782. doi: $10.1037 / \mathrm{a} 0032985$

Greenfield, B. L., Venner, K. L., Kelly, J. F., Slaymaker, V., \& Bryan, A. D. (2012). The impact of depression on abstinence self-efficacy and substance use outcomes among emerging adults in residential treatment. Psychology of Addictive Behaviors, 26, 246-254. doi:10.1037/ a0026917

Horsfall, J., Clearly, M., Hunt, G.E, \& Walter, G. (2009). Psychosocial treatments for people with co-occurring severe mental illnesses and substance use disorders (dual diagnosis): a review of empirical evidence. Harvard Review of Psychiatry, 17(1), 24-34. doi:10.1080/ 10673220902724599.

Iarussi, M. H., Tyler, J. M., Littlebear, S., \& Hinkle, M. S. (2013). Integrating motivational interviewing into a basic counseling skills course to enhance counseling self-efficacy. Professional Counselor, 3 (3), 161-174.

Luszczynska, A., Benight, C. C., \& Cieslak, R. (2009). Self-efficacy and health-related outcomes of collective trauma: A systematic review. European Psychologist, 14, 51-62. doi:10.1027/1016-9040.14.1.51

Lyne, J., O’Donoghue, B., Clancy, M., \& O'Gara, C. (2011). Comorbid psychiatric diagnoses among individuals presenting to an addiction treatment program for alcohol dependence. Substance Use \& Misuse, 46, 351-358. doi:10.3109/10826081003754757

Mason, S. J., Deane, F. P., Kelly, P. J., \& Crowe, T. P. (2009). Do spirituality and religiosity help in the management of cravings in substance abuse treatment? Substance Use \& Misuse, 44(13). Retrieved from http://informahealthcare.com/loi/sum/.

Miller, W. R., \& Rollnick, S. (2013). Motivational interviewing: Helping people change (3rd ed.). New York, NY: Guilford Press.
Najavits, L. M., \& Hien, D. (2013). Helping vulnerable populations: A comprehensive review of the treatment outcome literature on substance use disorder and PTSD. Journal of Clinical Psychology, 69(5), 433-479.

Patterson, D. A., Wolf, S., \& Buckingham, S. L. (2010). Does motivational interviewing stages of change increase treatment retention among persons who are alcohol and other drug dependent and HIV infected? Journal of HIV/AIDS \& Social Services, 9, 45-57. doi:10.1080/ 15381500903584346

Prochaska, J. O., Norcross, J. C., \& DiClemente, C. C. (2013). Applying the stages of change. Psychotherapy in Australia, 19(2), 10-15.

Stein, J. A., Zane, J. I., \& Grella, C. E. (2012). Impact of abstinence selfefficacy and treatment services on physical health-related behaviors and problems among dually diagnosed patients. Journal of Dual Diagnoses, 8, 64-73. doi:10.1080/15504263.2012.647470

Sterling, S., Chi, F., \& Hinman, A. (2011). Integrating care for people with co-occurring alcohol and other drug, medical, and mental health conditions. Alcohol Research \& Health, 33(4), 338-349. Retrieved from http://pubs.niaaa.nih.gov/publications/arh341/toc34_1.htmhttp:// pubs.niaaa.nih.gov/publications/arh341/toc34_1.htm.

Substance Abuse and Mental Health Services Administration, Center for Behavioral Health Statistics and Quality. (2014, September 11). The N-SSATS Report: Recovery services provided by substance abuse treatment facilities in the United States. Rockville, MD.

Tate, S. R., Mrnak-Meyer, J., Shriver, C. L., Atkinson, J. H., Robinson, S. K., \& Brown, S. A. (2011). Predictors of treatment retention for substance-dependent adults with co-occurring depression. The American Journal on Addictions, 20, 357-365. doi:10.1111/ j.1521.0391.2011.00137.x

United States Department of Substance Abuse and Mental Health Administration (SAMHSA) (2011). http://www.samhsa.gov/data/ sites/default/files/2011MHFDT/2k11MHFR/Web/NSDUHmhfr2011. htm\#Ch4

United States Department of Substance Abuse and Mental Health Administration (SAMHSA) (2014). Findings reported on adults who hold a SMI or AMI. Retrieved from http://samhsa.gov/data/2k14/ NSDUH170/sr170-mental-illness state-estimates

Westra, H. A., Aviram, A., Connors, L., Kertes, A., \& Ahmed, M. (2012). Therapist emotional reactions and client resistance in cognitive behavioral therapy. Psychotherapy, 49, 163-172. doi:10.1037/a0023200

Westra, H. A., Aviram, A., \& Doell, F. K. (2011). Extending motivational interviewing to the treatment of major mental health problems: Current directions and evidence. Canadian Journal of Psychiatry, 56 (11), 643-650. Retrieved from http://publications.cpa-apc.org/browse/ sections/0.

Wierzbicki, M., \& Pekarik, G. (1993). A meta-analysis of psychotherapy dropout. Professional Psychology: Research and Practice, 24(2), 190195.

Windsor, L. C., Jemal, A., \& Alessi, E. J. (2015). Cognitive behavioral therapy: A meta-analysis of race and substance use outcomes. Cultural Diversity and Ethnic Minority Psychology, 21(2), 300-313.

Winstanley, E. L., Gay, J., Roberts, L., Moseley, J., Hall, O., Beeghly, B., \& Somoza, E. (2012)

Wolff, N., Frueh, B. C., Shi, J., \& Schumann, B. E. (2012). Effectiveness of cognitive-behavioral trauma treatment for incarcerated women with mental illnesses and substance abuse disorders. Journal of Anxiety Disorders, 26(7), 703-710. 